\title{
The Anatomy Table - Is it the Future Learning Tool for Regional Anaesthesiologists?
}

\author{
Rajkumar Elanjeran ${ }^{1}$, Anitha Ramkumar ${ }^{1}$, Sandeep Ganni ${ }^{2}$ \\ ${ }^{1}$ Department of Emergency Medicine, GSL Medical College and General Hospital, Rajahmundry, Andhra Pradesh, India. \\ ${ }^{2}$ Managing Director, GSL Educational Society, Rajahmundry, Andhra Pradesh, India.
}

\begin{abstract}
Cadaveric dissection has been the main stay of anatomy training for regional anaesthesia over the years. Advent of advance visualisation hardware and software has revolutionised anatomy teaching and it is only a matter of time before this technology transcends into regional anaesthesia training. This article demonstrates the innumerable capabilities of virtual dissection table using one specific use case- the supraclavicular approach to the brachial plexus block.
\end{abstract}

Keyword: Simulation, Virtual dissection, Virtual anatomy

\section{Introduction}

Anatomy has been a bedrock of medical education for hundreds of years. The advent of modern technology has disrupted many traditional practices in clinical education, but anatomy has survived the most demanding pedagogic test-time.

The practice of regional anaesthesia is inconceivable without sound knowledge of the functional regional anatomy. Introduction of ultrasound in the practice of regional anaesthesia has further clarified the relationship of the needle and the nerve and the dynamics of local anaesthetic spread.

Regardless of the method of neuro localization, a thorough understanding of the surface anatomy and underlying three-dimensional structures is critical for providing a safe and successful approach.

Cadaveric dissection has been at the forefront of Anatomical training and is indispensable, but repetitive practice is not a viable plan of action for continuous onsite training given the logistical constraints behind procuring fresh frozen cadavers, especially when you're not located in a medical college.

Enter the Anatomy Table - A technological milestone bar none. Although there are several products in the market, for the purpose of this paper, we will be referring to the most prominent and widely used product - The Anatomage table. The Anatomage table features full high-resolution 3D real human anatomy learning cases that can be interacted via touch. By utilizing a touch interactive screen and sophisticated 3D anatomy visualization software, students can rotate the virtual body, make multiple cuts, undo any cut instantly, and create notes. cut it in any direction. The Table offers unique interactive dissection tools with thousands of annotations for both male and female cadavers. They can explore the body by selecting different structures or locating structures from a list of names.

\section{Anatomage Features}

The several features of the app as illustrated on the home screen of the table are detailed below. This will give you a quick overview of the vast capabilities of the table. The user interface has a short learning curve and is very intuitive to

Address of Correspondence:

Dr. Rajkumar Elanjeran

Manager, Clinical Lead of Simulation, and Consultant Emergence Physician,

GSL Medical College and General Hospital, Rajahmundry, Andhra Pradesh, India.

E-mail: seran50.raj@gmail.com

Submitted: 9 March 2021; Reviewed: 23 March 2021; Accepted: 24 April 2021; Published: 10 July 2021

DOI: $10.13107 /$ ijra.2021.v02i02.038 | www.ijrajournal.com |

This is an Open Access article distributed under the terms of the Creative Commons Attribution Non-Commercial-Share Alike 4.0 License

(http://creativecommons.org/licenses/by-nc-sa/4.0) which allows others to remix, tweak, and build upon the work non-commercially as long as appropriate credit is given and the new creation are licensed under the identical terms. 


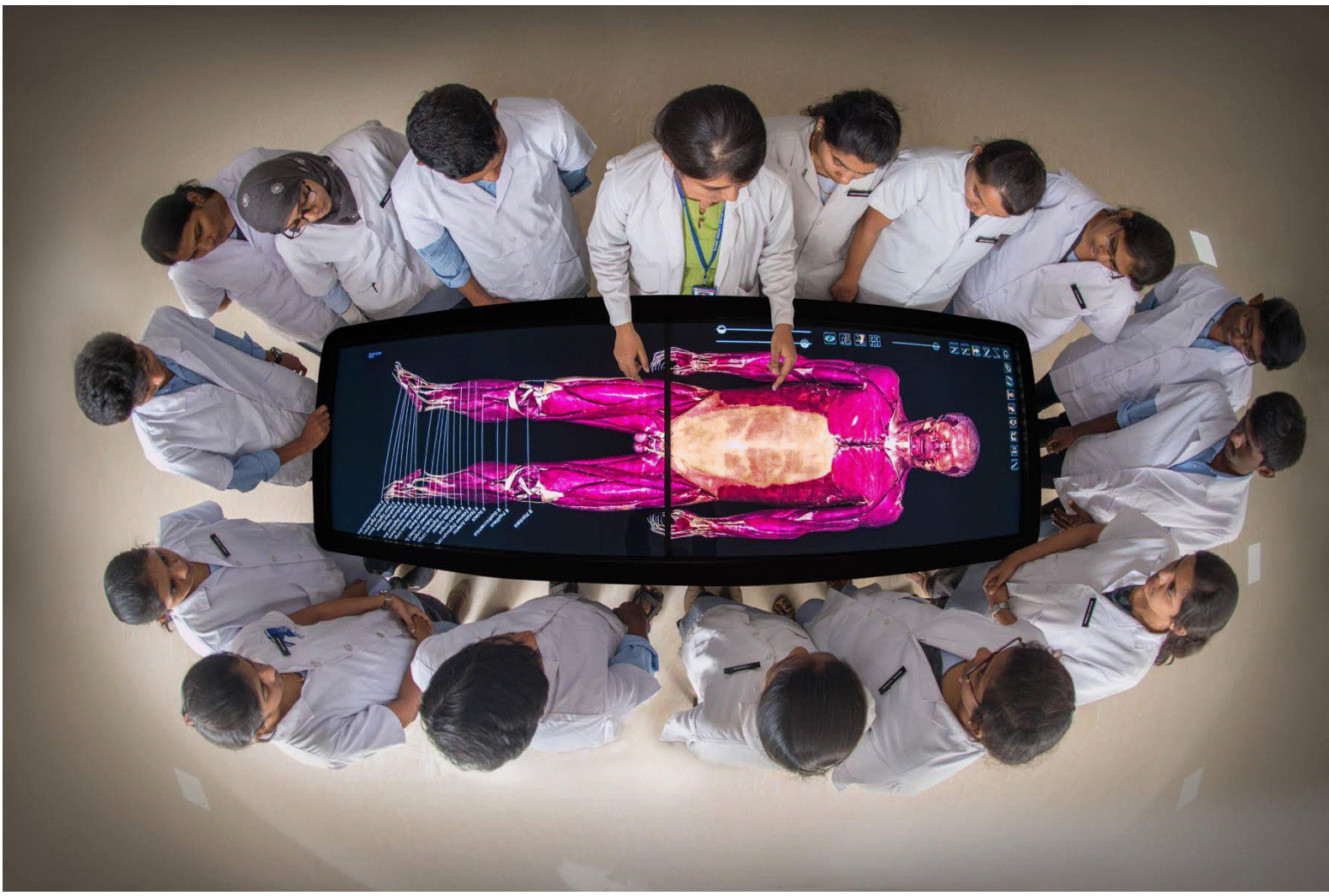

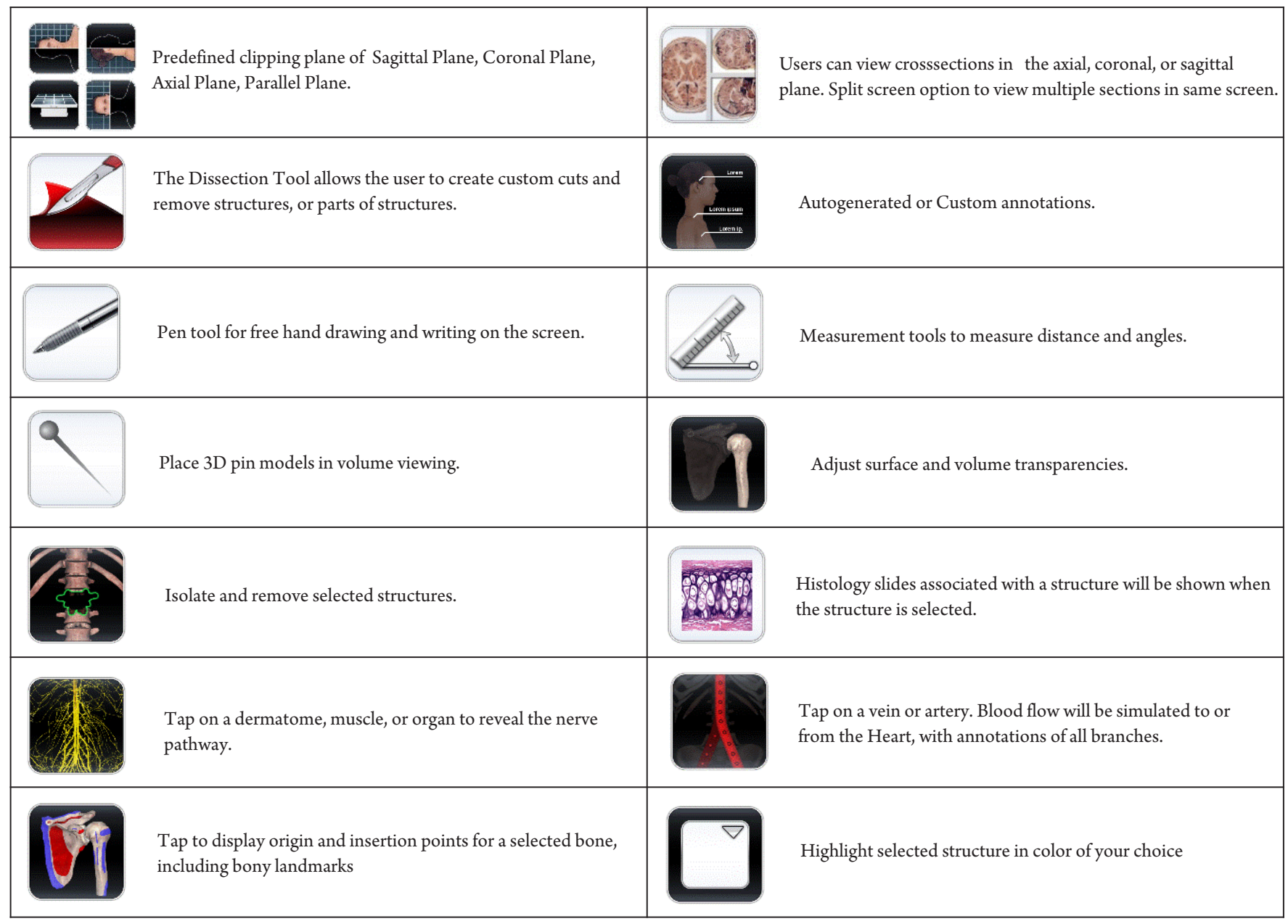

\section{Anatomage Features}




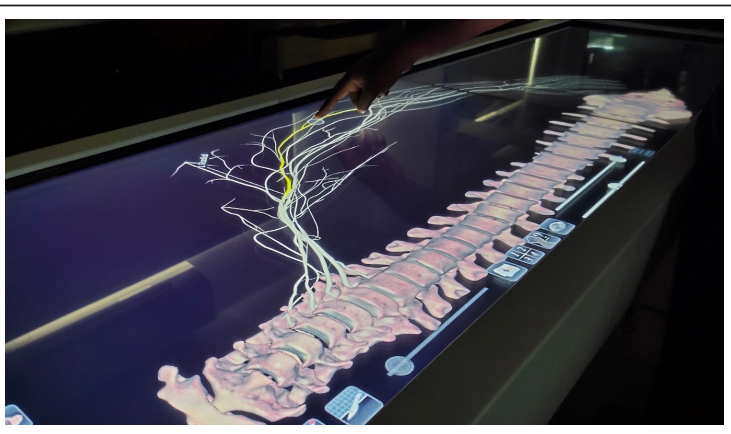

Figure 1: The above image illustrates the brachial plexus in its exact anatomical position as you would see in the human body. The left radial nerve is being highlighted as an example
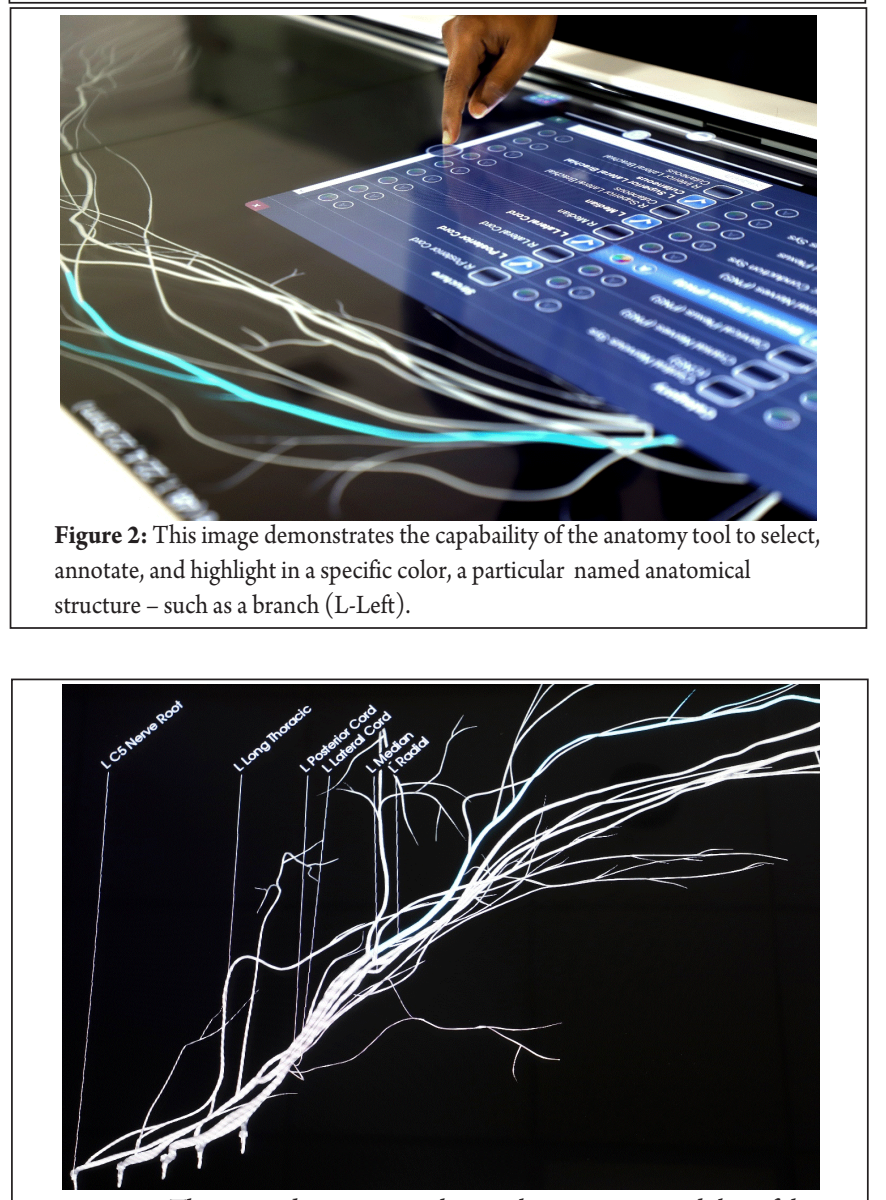

Figure 3: The image demonstrates the serial annotation capability of the anatomy table (L-Left)

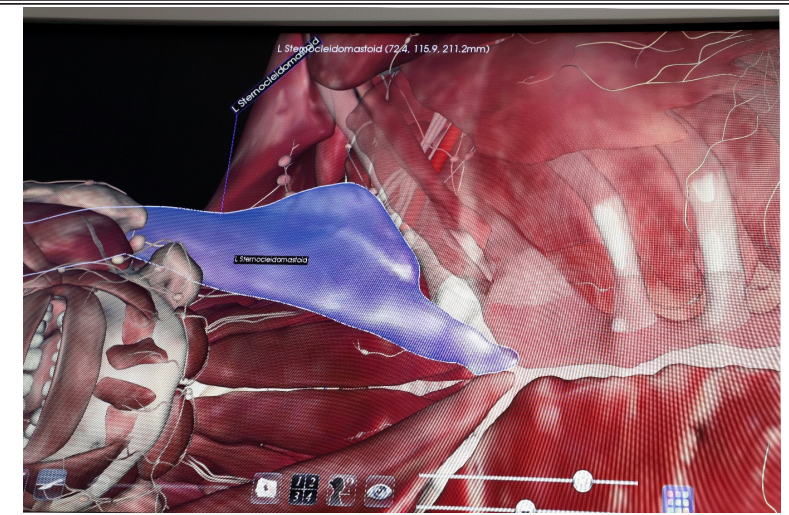

Figure 4: This image demonstrates how the skin and subcutaneous tissue can be removed and the muscle highlightes in a different colot (purple) to highlight surface landmark correlation (L-Left). even the most amateur of technology enthusiasts.

To illustrate an actual use case for the Anatomy table in regional anaesthesia education, we shall be using an example of a supraclavicular approach of the Brachial plexus block . The supraclavicular approach is the cornerstone of distal upper extremity regional anaesthesia, and is used extensively with a very low rate of complications.

\section{Supraclavicular brachial plexus block}

The brachial plexus, as any plexus goes, is one of the most anatomically complex structures known. The brachial plexus can be a very challenging topic to understand due to its complex origin, branching, and relations and students often get lost while reading the textbooks. It is for this very reason that most educational tools for teaching brachial plexus are modelled around using a schematic interpretation rather than the actual and exact anatomical structure of the brachial plexus. This proves as an additional challenge for the regional anaesthetist, as a schematic interpretation has little to no relevance when considering anatomical correlates. In our experience, whenever a student is shown the true isolated anatomically structure of the brachial plexus on our table, the immediate response is one of confusion and disbelief. (Figure 1 and 2 )

It is thus prudent that regional anaesthesia be taught using the actual anatomical structure and the anatomy table is the perfect and only unique tool which can enable this form of teaching. A specific nerve can be highlighted using a different colour and traced back along its root value. (Figure 3)

\section{Anatomical correlations}

1. Surface landmarks- the lateral (posterior) border of the SCM muscle is identified and followed distally to the point where it meets the clavicle (Figure 4) A margin of safety is established approximately $2.5 \mathrm{~cm}$ laterally to the insertion, parasagitally. The needle is first introduced perpendicular to the skin and is then turned and advanced parallel to the midline, about one finger breadth above the clavicle.

2. Osteological correlation- The brachial plexus passes over the first rib in the supraclavicular fossa and this can be easily elicited on the table, by adding in the skeletal system in addition to the brachial plexus, and chose which bones to add or remove, which bones to highlight and which bones to make transparent.

In this image (Figure 5). The first rib is highlighted in a different colour, where as the clavicle has been made transparent, to better appreciate the brachial plexus. This 


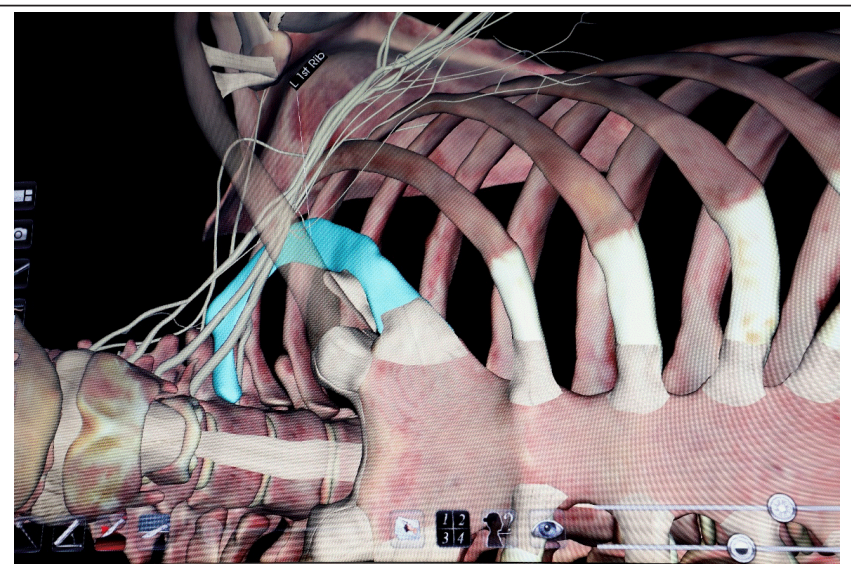

Figure 5: Left first rib highlighted in a different color under the clavicle (which has been made transparent) to demonstrate osteological correlates (L-Left).

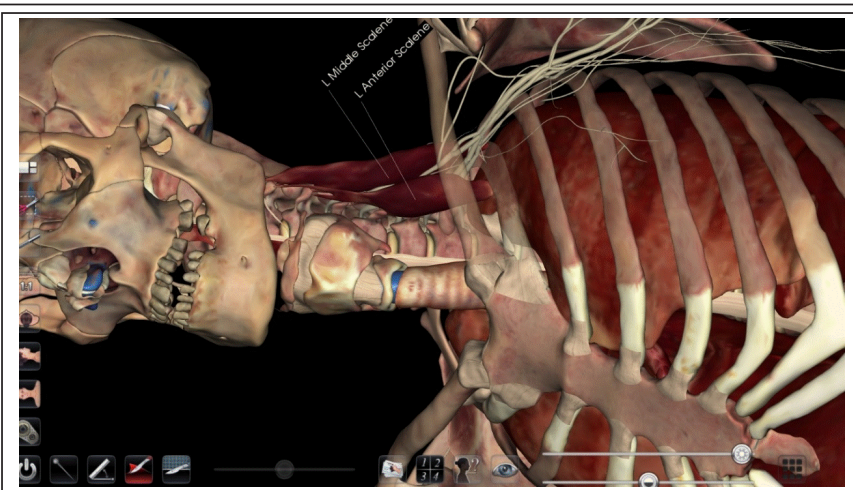

Figure 6: The muscular correlates of the brachial plexus at the supraclavicular level - namely Left Middle scalene and Left Anterior Scalene (L-Left).

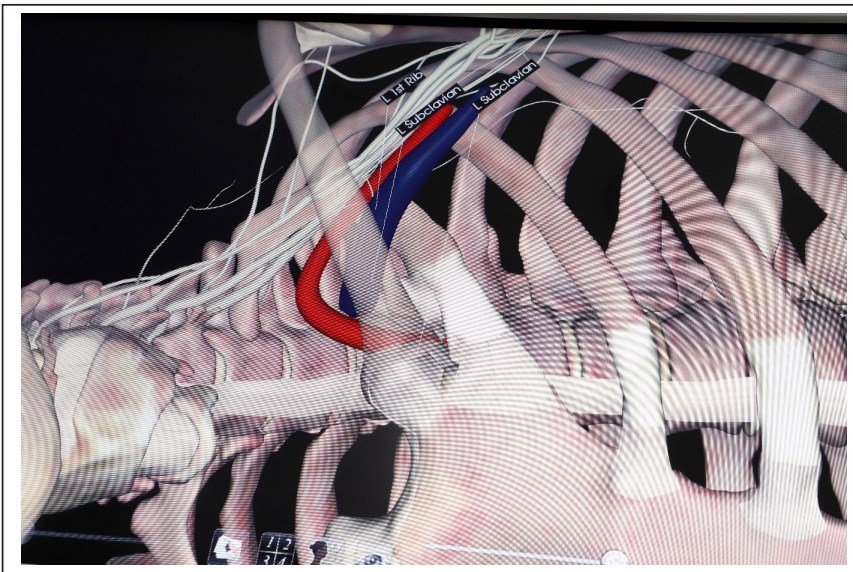

Figure 7: The clavicle has been made transparent and the left subclavian artery and vein have been isolated and annotated (L-Left).

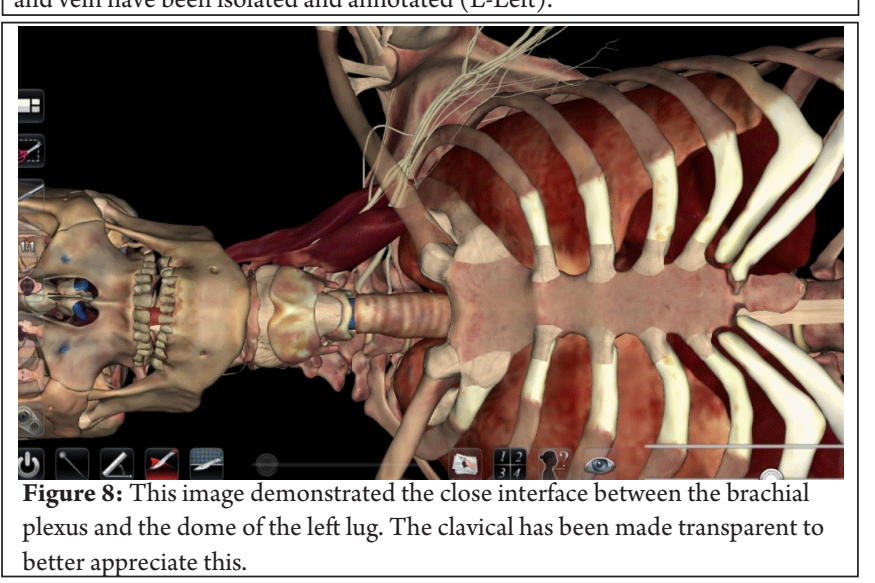

level of customization is only limited by the teacher's and student's imagination. Despite the customisation, the student still witnesses a true anatomy image rather than an animation or computer generated graphic.

\section{Muscular Correlation}

The five roots originating from the ventral divisions of $\mathrm{C5}$ through $\mathrm{T} 1$ form the brachial plexus The roots lie between the anterior and middle scalene muscles The five roots converge toward one another to form three trunks-upper, middle, and lower-which are stacked one on top of the other as they traverse the triangular interscalene groove, formed between the anterior and middle scalene muscles. The brachial plexus, in the interscalene grove is stacked more vertically, and as it passes underneath the clavicle, it has to flatten out and and the orientation becomes more horizontal or oblique, making it appear wide. As show in the figure 6, specific muscles can be selected to be viewed while surrounding structures can be removed. The Clavicle has been made transparent to view the brachial plexus passing underneath.

\section{Vascular correlation}

The subclavian artery accompanies the brachial plexus in the interscalene groove anterior to the lower trunk, as it rests on the first rib. (Figure 7)

\section{Organ Correlates}

Pneumothorax is a significant complication of the supraclavicular block. The pleura can potentially be injured in two places during a supraclavicular block; the pleural dome and the first intercostal space. The pleural dome is the apex of the parietal pleura, circumscribed by the first rib. The first rib is a short, broad, and flattened bone shaped like the letter $\mathrm{C}$. It's medial border forms the outer boundary of the pleural dome. The anterior scalene, by inserting in this border of the first rib, comes in contact medially with the pleural dome. There is no pleural dome lateral to the anterior scalene muscle. The first intercostal space on the other hand, is for the most part infraclavicular and consequently should not be reached when a supraclavicular block is properly performed. (Figure 8)

\section{Axial Sections}

Axial sections provide the learners with a complete understanding of what structures they will encounter as they penetrate the skin with the needle and reach further. In addition, The ability to use a specific axial section will help the learner understand true anatomical correlates for Ultrasound guided regional anaesthesia. 


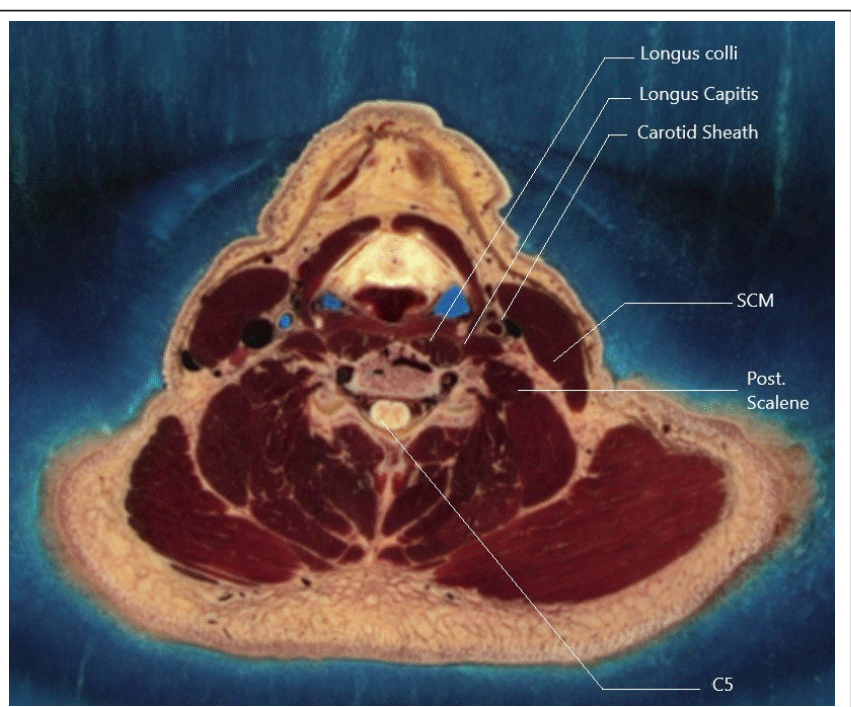

Figure 9: Axial section at the C5 Level demonstrating musculular correlates.

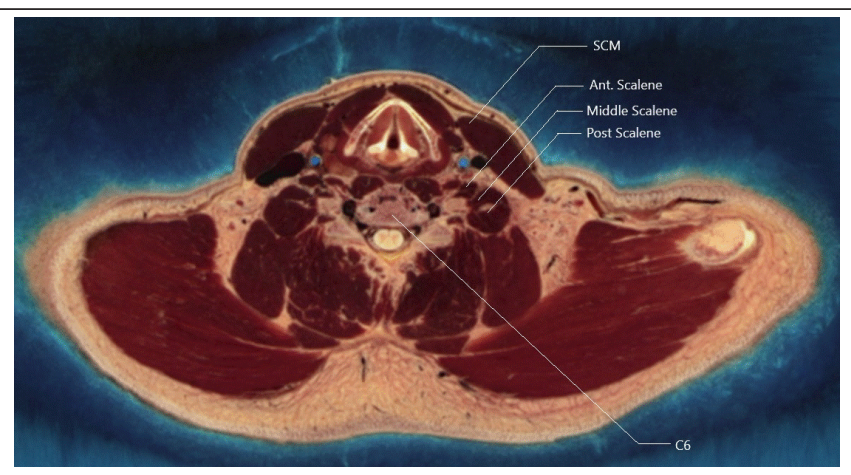

Figure 10: Axial section at C6 level demonstrating muscular correlates.

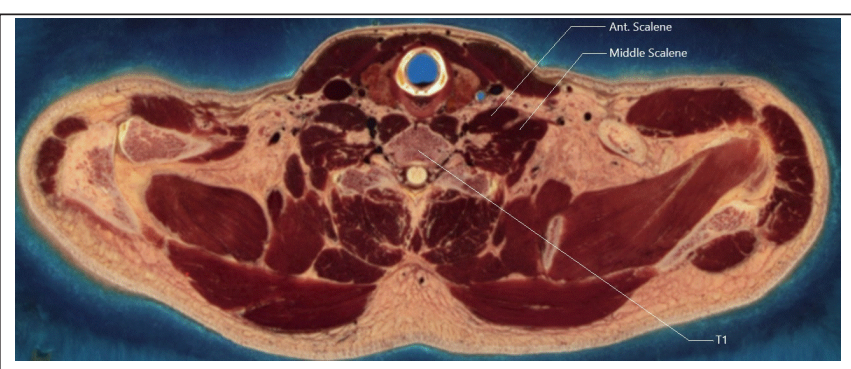

Figure 11: Axial section at T1 level.

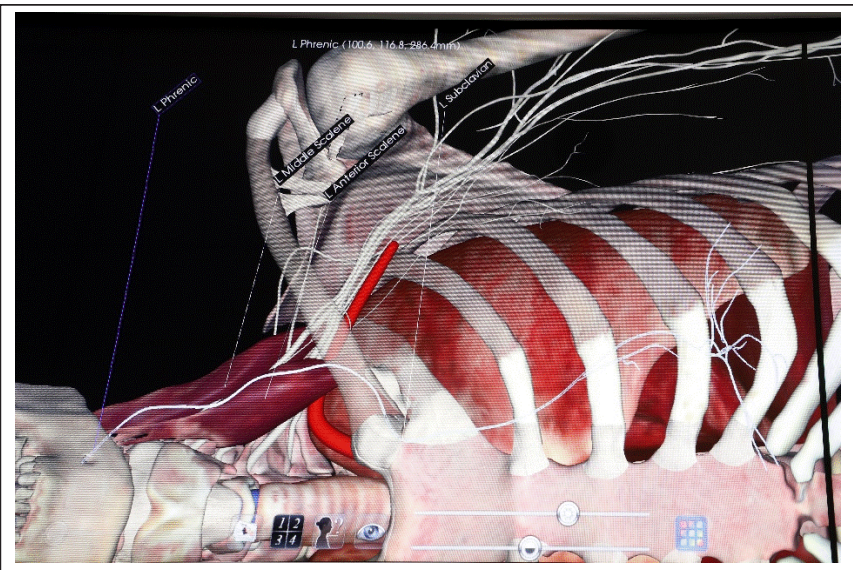

Figure 12: The close positioning of the Left phrenic nerve in relation to the brachial plexus at the supraclavicular level (L-Left).
The software has the ability to render axial sections which are actual cadaveric sections.

\section{Neurovascular complications}

Phrenic nerve block is one of the complications encountered with the surpaclavicular block (Figure 12) Although the incidence is much lower than that of interscalene block, but the incidence is still significant.

The plexus itself is sometimes directly innervated by the dorsal scapular artery, and in close proximity to other vessels namely the sublcavian artery and vein, transverse cervical artery, External Jugular vein, and suprscapular vein. It is thus pertinent to aspirate frequently as you enter the supraclavicular fossa.

\section{Advantages of anatomage table}

1. Advanced educational tool and technical showcaseThe accuracy of real human anatomy and quantity of clinical cases are unique aspects of the Anatomage Table. The Table includes ultra-high quality (UHQ) visualization for students to view photorealistic anatomical structures. This draws attention from visitors as well as well as students and faculty. The Table has quickly become the technological centerpiece at your institution.

2. Clinical care review- Beyond anatomy education, the Table's application extends to clinical planning and consultation. The Anatomage Table is FDA cleared for use in assisting medical diagnosis. It can be utilized as a powerful radiology workstation and as a valuable tool for surgical case review, patient consultation, and medical research.

3. Cost reduction- Unlike cadavers, the Anatomage Table does not require ventilation infrastructure, embalming equipment, personnel, or storage. The contents are reusable, so there are no recurring acquisition costs. The product will save significant costs over the long term.

4. Clean and safe- The Anatomage Table offers a high quality lab experience without any chemicals. There are no possibilities of leaks, no environmental concerns, and no additional ventilation requirements. The product provides headache free lab sessions.

\section{Limitations}

1. Cost- The table costs a very high premium and is not something that you will come by easily. It takes a specific and ambitious financial motivation by an institute to invest so much money in a single product because the return of investment is next to non existent.

2. Is this future proof- The anatomy table is, in its present state, the most advanced market ready anatomy teaching tool. But we are currently in the generation of wearable 
devices such a Microsoft Holo lens and other VR players which greatly increase the scope and scale of learning anatomy. Holo lens, for instance, has app already for anatomical models that can be projected Three dimensionally in your own visual space with seamless integration. Scalability of these devices are a huge challenge and it remains to be seen if these will replace the anatomy table.

3. Immobile joint movements- The virtual cadaver can be rotated and viewed in any plane, but the joint mobility is fixed and you cannot flex or extend or move a body part to get a different view.

4. Adjunct or replacement? - The anatomy table will still serve only as an adjunct to actual cadaver teaching. The reasons for this are manifold and will require an entire article of its own. Anyone who's dissected a cadaver will understand the importance of the touch and feel of dissection, and this is why the table can never replace cadaveric teaching.

5. Dye injection techniques- Cadaveric teaching enables the use of dyes to demonstrate injection spread dynamically. This, however, is not applicable to the anatomy table.

6. Sonography- The anatomy table, despite being an FDA approved PACS viewer, is still not equipped for ultrasound correlation.
7. Limited deviations- The table comes pre-loaded with a fixed number of cadavers. Despite the infinite learning possibilities in this table, it is still limited to these pre-loaded cadavers. The pre-loaded data cannot be modified to teach abnormalities or anatomical deviations.

\section{Conclusion}

The Anatomage Table's powerful content creation tools and demonstration capabilities gives users a complete platform for medical education. Numerous institutions such as medical universities, undergraduate programs, and school districts use the Table as a complete lab alternative. The Table serves as a valuable tool for clinical planning and accurately visualize internal and surface anatomy in $3 \mathrm{D}$ for clinical training. Functional regional anaesthesia is not just about muscle memory, but also the ability to visualize and plan your approach and execution of the block. The Anatomy table is second to none when it comes to perfecting and impressing the best form of visual memory into the learners mind.

Declaration of patient consent: The authors certify that they have obtained all appropriate patient consent forms. In the form, the patient has given his consent for his images and other clinical information to be reported in the Journal. The patient understands that his name and initials will not be published, and due efforts will be made to conceal his identity, but anonymity cannot be guaranteed.

Conflict of interest: Nil Source of support: None

\section{References}

1. Fyfe, Sue \& Fyfe, Georgina \& Dye, Danielle \& Radley-Crabb, Hannah. (2018). The Anatomage table: Differences in student ratings of usefulness from first implementation to established use.

2. Alessandro Stecco, Francesca Boccafoschi, Zeno Falaschi, Giulio Mazzucca, Andrea Carisio, Simone Bor, Irene Valente, Sergio Cavalieri, Alessandro Carriero,. Virtual dissection table in diagnosis and classification of Le Fort fractures: A retrospective study of feasibility, Translational Research in Anatomy, Volume 18, 2020, 100060, ISSN 2214-854X.

3. Taoum, Alexandre \& Sadqi, Rihab \& Zidi, Mustapha \& Tassigny, Alexandra \& Megdiche, Kawtar \& Ngote, Nabil. (2019). On the Use of Anatomage Table as Diagnostic Tool. 13.20-25.
4. J. Brown, S. Stonelake, W. Anderson, M. Abdulla, C. Toms, A. Farfus, J. Wilton, Medical student perception of anatomage - A 3D interactive anatomy dissection table, International Journal of Surgery, Volume 23, supplement 1,2015, Pages S17-S18.

5. Smith, K.E., Ruholl, H.O. and Gopalan, C. (2019), Utilization of Anatomage Table Technology Enhances Knowledge, Comprehension, and Application of Human Anatomy and Physiology in Multiple Settings. The FASEB Journal, 33: 598.19, 598.19.

How to cite this article: Elanjeran R, Ramkumar A, Ganni S | The Anatomy Table - Is it the Future Learning Tool for Regional Anaesthesiologists? | International Journal of Regional Anaesthesia | July-December 2021; $2(2)$ : 111 -116. 